\title{
Social fears and social phobia in a community sample of adolescents and young adults: prevalence, risk factors and co-morbidity
}

\author{
H.-U. WITTCHEN, ${ }^{1}$ M. B. STEIN AND R. C. KESSLER \\ From the Max Planck Institute of Psychiatry, Clinical Psychology and Epidemiology, Munich, Germany
}

\begin{abstract}
Background. The paper describes prevalence, impairments, patterns of co-morbidity and other correlates of DSM-IV social phobia in adolescents and young adults, separating generalized and non-generalized social phobics.

Methods. Data are derived from the baseline investigation of the Early Developmental Stages of Psychopathology Study (EDSP), a prospective longitudinal community study of 3021 subjects, aged 14-24. Diagnoses were based on the DSM-IV algorithms of an expanded version of the Composite International Diagnostic Interview.
\end{abstract}

Results. Lifetime prevalence of DSM-IV/CIDI social phobia was $9.5 \%$ in females and $4.9 \%$ in males, with about one-third being classified as generalized social phobics. Twelve-month prevalence was only slightly lower, indicating considerable persistence. Respondents with generalized social phobia reported an earlier age of onset, higher symptom persistence, more co-morbidity, more severe impairments, higher treatment rates and indicated more frequently a parental history of mental disorders than respondents with non-generalized social phobia.

Conclusions. History of DSM-IV social phobia was found to be quite prevalent in 14-24 year-olds. The generalized subtype of social phobia was found to have different correlates and to be considerably more persistent, impairing and co-morbid than non-generalized social phobia. Although generalized social phobics are more likely than non-generalized social phobics to receive mental health treatments, the treatment rate in this sample was low despite the fact that mental health services are free in Germany.

\section{INTRODUCTION}

In the past two decades the prevalence of social phobia has been studied in several community studies throughout the world. Most of the earlier studies were based on DSM-III criteria assessed with the Diagnostic Interview Schedule (DIS) (Robins et al. 1981). The Epidemiologic Catchment Area (ECA) programme found the lifetime (LT) prevalence of DSM-III social phobia to be

1 Address for correspondence: Dr Hans-Ulrich Wittchen, Max Planck Institute of Psychiatry, Clinical Psychology and Epidemiology, Kraepelinstrasse 2, 80804 München, Germany.
$2.8 \%$ (Regier et al. 1990). Similar DIS/DSM-III estimates were obtained in community surveys carried out in Munich (2.5\%) (Wittchen et al. 1992), Edmonton (1.7\%) (Bland et al. 1988), Zurich (3.2\%) (Angst \& Dobler-Mikola, 1985) and New Zealand (3.9\%) (Wells et al. 1989). These prevalence could be regarded as fairly conservative estimates due to the fact that the version of the DIS used in these surveys assessed social fears as part of the simple phobia section, covering only a limited range of social fear situations (Davidson et al. 1993; Walker \& Stein, 1995; Magee et al. 1996; Wittchen \& Beloch, 1996). It is noteworthy that the DIS/ 
DSM-III rates are also considerably lower than those found in previous non-DIS studies by Marks (1979; 8\%), Bryant \& Towers (1974; $10 \%)$ and DiNardo et al. $(1983 ; 13.3 \%)$.

This 'underestimation' problem, which possibly was due to the lack of precision in DSM-III, has been corrected in the successor to the DIS, the World Health Organization's Composite International Diagnostic Interview (CIDI) (WHO, 1990), by developing a separate social phobia module according to the diagnostic criteria of DSM-III-R and the ICD-10, Diagnostic Criteria for Research. This module evaluates more types of social fears than the original DIS. Community epidemiological surveys using the CIDI have obtained considerably higher estimates of social phobia than all earlier studies, including a $13.3 \%$ lifetime DSM-III-R prevalence in the US-National Comorbidity Survey (Magee et al. 1996) and a 16.1\% lifetime ICD-10 prevalence in Basel, Switzerland (Wacker et al. 1992). Although some part of these higher prevalence estimates than in the earlier DIS/DSM-III surveys might be due to the expanded DSM-III-R and ICD-10 criteria, differences in sample composition or field procedures and the much more comprehensive assessment of social fears are likely to be responsible for most of the increase (Stein et al. 1994; Wittchen \& Beloch, 1996).

Despite the substantial differences in estimated prevalence, there is good agreement across studies on risk factors for social phobia in adults. Rates of social phobia are consistently found to be slightly higher among women than men, higher in younger as compared to older age cohorts, and inversely associated with socioeconomic status (Bourdon et al. 1988; Bourdon, 1993; Magee et al. 1996). There is also agreement across studies that social phobia usually has its onset in childhood or adolescence, usually goes untreated (Burns, 1980; Ross, 1993; Schneier et al. 1992; Magee et al. 1996), and is associated with poor school and work performance, school dropout, unemployment, and alcohol abuse (Mullaney \& Trippet, 1979; Liebowitz et al. 1985; Davidson et al. 1993; Wittchen \& Beloch 1996). There is also consistent evidence that social phobics have a higher risk than people with no social phobia of other anxiety disorders, major depression and substance use disorders (Merikangas et al. 1996). These other disorders seem to occur mostly secondary to social phobia. However, this conclusion should be interpreted with caution due to the retrospective assessment strategies used in these studies. Other potentially important vulnerability and risk factors for the development of social phobia, like familial liability (Stein et al. 1998) and childhood 'behavioral inhibition' (Biederman et al. 1990; Rosenbaum et al. 1992) have not yet been studied in any epidemiological study.

Little is known about the severity and impairments due to social phobia in community samples (Magee et al. 1996). The only assessment of impairment in most studies was a single yes-no question about whether social phobia interfered a lot with life and activities. More fine-grained assessments of impairment have not been made. Neither has there been an attempt to sort out the impairment due to social phobias from that due to co-morbid conditions (Wittchen, 1996). Furthermore, few epidemiological data exist concerning the frequency of subtypes of social phobia (Stein et al. 1994, 1996). No epidemiological study up to now has investigated the DSM-IV subtype of generalized social phobia.

This report provides information about the lifetime and 12-month prevalences of social fears and DSM-IV social phobia in a community sample of adolescents and young adults aged 14-24. This emphasis on fairly young respondents allows a more powerful age-group-specific analysis of risk factors, impairments and correlates of social phobia than in previous studies. We emphasize differences between generalized and non-generalized social phobia in symptoms, severity, co-morbidity, correlates and disorderspecific impairments and disabilities and will further reports some findings about the association of parental psychopathology and behavioural inhibition with social phobia.

\section{METHOD}

The data were collected as part of the baseline investigation of the Early Developmental Stages of Psychopathology (EDSP) study. The EDSP is a research programme funded by the German Ministry of Research and Technology, designed to collect data on the prevalence, risk factors, co-morbidity and course of mental disorders in 
a community sample of 3021 adolescents and young adults. The study consists of a baseline survey and two follow-up surveys at approximately 15 and 30 months after baseline and a separate family genetic investigation of the parents of respondents. A full description of the study is presented elsewhere and thus the methods will be discussed only briefly here (Wittchen et al. 1998a).

\section{Sample}

The EDSP sample was drawn from the 1994 official population register of all residents in metropolitan Munich. All registrants who were expected to be 14-24 years-of-age during the first half of 1995 were eligible for selection. Because the study was designed as a longitudinal investigation with special interest in the onset and early development of mental disorders, 14-15 year-olds were oversampled. From 4263 individuals eligible for the study, a total of 3021 interviews were completed resulting in a response rate of $71 \%$. To account for differential sampling probabilities and non-response, the data reported in this paper were weighted to match the age-sex distribution of the sampling frame.

Approximately one-third of the sample was attending at the time of study or had attended Gymnasium (secondary education between ages of 10 to 19 which prepares students for entrance to university) and another third university. Lower school educational status was reported by $11 \%$ (Hauptschule, or mandatory basic school) and intermediate school by $18.4 \%$ (Realschule, or intermediate advanced school between Gymnasium and Hauptschule allowing qualification for specific university curricula). Consistent with the mandatory schooling laws, only $0.5 \%$ of respondents dropped out of school without any degree. Only $22.6 \%$ of all study participants were in the work force at the time of interview and another $4.9 \%$ were registered as being unemployed. The majority of respondents $(64 \%)$ were living with their parents and only a small number were married $(3 \%)$ at the time of interview. The vast majority $(60 \cdot 2 \%)$ of respondents were classified into middle class.

\section{Measures}

Psychopathological and diagnostic assessments were based on the computer-assisted personal interview (CAPI) version of the Munich-Composite International Diagnostic Interview (MCIDI) (Wittchen \& Pfister, 1997). The M-CIDI is a modified version of the World Health Organization (WHO) CIDI, version 1.2 supplemented by questions to cover DSM-IV and ICD-10 criteria (Wittchen \& Pfister, 1997). The M-CIDI allows for the assessment of symptoms, syndromes and diagnoses of 48 mental disorders along with information about onset, duration, and severity. The CAPI version of the M-CIDI was supplemented by a separate respondents' booklet that included several scales and questionnaires that are of importance for the longitudinal component of our study. The booklet also included cognitive aids to assist the respondent in dating symptom onset and recency, answering complicated symptom questions, and identifying course patterns. Diagnostic findings reported in this paper are based on the CIDI/DSM-IV algorithms (Wittchen \& Pfister, 1997) without using the DSM-IV hierarchy rules. The mean duration for completing the computerized (CAPI) M-CIDI, including questionnaires, was 77 min.

Because the M-CIDI social phobia module contains more detailed questions for assessing DSM-IV criteria than previous versions, the assessment procedure and the algorithms will be described briefly. The section begins with a stem question to sensitize the respondent to subsequent seven questions about specific social fear situations. Before reading this stem question the respondent was instructed to open a respondent booklet to review visually seven situational clusters of situations along with more detailed examples. These seven situations are identical to the standard CIDI and were coded for use in the diagnostic algorithm. This visual aid presented up to three more specific examples for each of the seven standard CIDI situational fear 'cluster' questions (i.e. when asking for 'ever having strong fears of attending social affairs, going to a party or meeting', the following additional situations, taken from various social phobia questionnaires, were additionally visually presented for subsequent coding: participating in small groups, going to a party, entering a room when others are waiting). Respondents were asked to give a concrete example for each item endorsed to allow for clarification by the interviewer and for later editing. After at least 
one fear situation was elicited, a subsequent series of nine questions was asked about cognitive elements of anxiety such as fear of blushing, fear of panic, fear of showing symptoms of anxiety, etc. DSM-IV Criterion B was assessed by instructing subjects to turn to a list of anxiety symptoms in the respondent booklet and to indicate all symptoms of anxiety occurring in such situations. Because DSM-IV requires that cued anxiety reactions occur almost invariably, the M-CIDI algorithm required at least two such symptoms occurring 'almost always' when thinking about, or when being exposed to such situations. Criterion $\mathrm{C}$ was assessed by asking the respondent whether they considered either the anxiety or the avoidance to be excessive or unreasonable. Criterion $\mathrm{D}$ was assessed by determining if the respondent frequently avoided the situations or, if not, endured the situations with distress. Criterion E was assessed by determining if respondents reported that: (a) the social fears or avoidance interfered a lot with at least one of three social role areas; (b) they were persistently upset for a period of at least 1 month; or $(c)$ they sought professional help for the fears. The persistence of social phobia (criterion F) by assessing the duration of symptoms and whether the phobic anxiety reactions occur at least mostly over this time period. The DSM-IV diagnostic exclusion G and $\mathrm{H}$ were assessed with specific probe questions to evaluate medical (including stuttering, speech defects, abnormal eating behaviours, mental disorders such as panic) or substance-use related syndromes. Throughout the paper, reported DSM-IV prevalence rates take into account the above mentioned exclusion criteria.

A diagnosis of social phobia, generalized subtype is assigned by the expanded M-CIDI diagnostic algorithm, whenever: (a) all diagnostic criteria were met; $(b)$ additionally, three or more of the overall six specific qualifying classes of social fear situations presented were reported; and $(c)$ the person acknowledged that fear and avoidance of these situations almost always were present since onset. It is noteworthy that the M-CIDI includes a seventh residual category of 'other' situations when respondents were in the centre of attention of others. The examples given to this item, however, were reviewed by the clinical editors and were then in most cases assigned to the six main classes of social fears. In terms of the critical threshold of number of situations the M-CIDI generalized phobia algorithm compares well to the procedure used by Manuzza et al. (1995). Our strategy requires at least three situational clusters out of a total of six, including test and performance fears; Manuzza et al. (1995) cases mostly had at least three interactional and frequently one additional performance fear out of a total of ten.

The diagnostic 1-week test-retest reliability of the M-CIDI social phobia module, investigated in a general population sample of 60 cases, had a kappa of 0.72 and slightly higher for the generalized subtype (kappa: 0.80) (Wittchen, 1994; Wittchen \& Pfister 1997; Wittchen et al. $1998 b$ ). Validity of the M-CIDI diagnoses of social phobia as compared to independent clinical consensus diagnoses by treating physicians in a sample of 68 randomly chosen patients had a kappa of 0.80 , a negative predictive value of 0.714 , and a positive predictive value of $1 \cdot 0$ (Reed et al. 1998).

\section{Additional measures}

Behavioural inhibition was assessed with the German version of the Retrospective SelfReport of Inhibition questionnaire (RSRI; Resnick et al. 1992). This questionnaire consists of a set of 30 questions reflecting several domains (i.e. sympathetic nervous system activity, fear of things, etc) to be answered retrospectively for the time as a child (school grades 1 to 6). Although the authors suggested a two-factor solution, preliminary psychometric investigations revealed only one main factor; thus for the present analysis subjects we will examine the role of high (as defined as a standard score of $z$ $<0.49)$ behavioural inhibition. Parental psychopathology was assessed by the M-CIDI family history module (Wittchen \& Pfister 1997), that consists of a series of 42 complex questions to assessing for the respondent father and mother as well as other family members indicators (sought professional help, noticed key symptoms, their persistence, etc.) for various types of mental disorders (anxiety, depressive somatoform, psychotic, substance use disorder). Since pilot-comparisons of findings from these questions with the results of an independent personal assessment of the respondents' 
(Wittchen et al. 1999) parents revealed only moderate agreement for specific diagnoses, this paper restricts the presentation to a dichotomous variable that was found to be sufficiently concordant with the findings of separate personal parents interviews to warrant investigation (kappa of 0.64): parental history of mental disorders as evidenced either by a known mental health treatment history or perceived problems in the areas of anxiety, depression, somatoform disorders. Work disability and productivity is indicated by number of days in the past month the person was completely unable (disability) to perform his social roles, or was at least significantly limited (productivity) because of his symptoms. Help-seeking and treatment information was assessed comprehensively including health insurance data; from this comprehensive data base, however, only data about social phobia-specific interventions by medical doctors and other mental health practitioners as well as mental health interventions for other reasons will be presented.

\section{Interviewer and field work}

The survey staff was highly experienced and consisted of 10 clinical interviewers and 25 professional health-research interviewers. All had extensive experience in diagnostic interviewing including the CIDI and in the developmental work of the M-CIDI. All interviewers received two full weeks of training followed by at least 10 practice interviews that were closely monitored by our staff. Immediately prior to the beginning of the study, an additional training session was conducted. Contact attempts were initiated by mail. A time and location for the interview were then established by phone. Most interviews took place in the home of the probands. Written and signed informed consent was obtained from all participants. To enhance participation, various special efforts were made during the study ranging from assigning different interviewers at different times to contact hard to reach respondents to financial incentives for participation.

\section{Analyses}

To account for differential sampling probabilities and non-response, the data reported in this paper were weighted by age and sex to match the distribution of the sampling frame.
All statistical analyses, except $\chi^{2}$-tests, were done using SAS software (SAS, 1996) and relative weights. To account for design effects introduced by using weighted data, all standard errors and statistical tests were estimated using SUDAAN (Shah et al. 1996).

\section{RESULTS}

\section{Lifetime (LT) and 12-month prevalence by age and gender}

Overall, $4.9 \%$ of males and $9 \cdot 5 \%$ of females aged 14-24 meet lifetime DSM-IV criteria for social phobia (Table 1). About one-third of lifetime cases meet study criteria for the generalized subtype. Overall, females have significantly higher lifetime and cross-sectional rates than males $(P<0 \cdot 001)$. Lifetime estimates for 14-17 year-olds are lower than those for 18-24 year-olds. The majority of lifetime cases also meet diagnostic criteria in the past 12 months. The persistence of generalized social phobia (males, $76.8 \%$; females, $90 \%$ ) from LT into the past 12-months is slightly higher than in isolated social phobia (males $59.5 \%$; females $69 \cdot 2 ; P=0 \cdot 03)$.

In an attempt to cover DSM-IV diagnostic exclusion criteria $G$ and $F$, we examined what proportion of social phobics might have developed their disorder as a result of substance use or of somatic illnesses and conditions. No case was identified with a suspicious substance factor that might be related to the onset of social phobia. However, two social phobics had at least mild chronic physical disablement (partial amputation of three fingers, noticeable limping due to early childhood accidents and subsequent operation) and four had visible physical defects (two cases of severe long-lasting acne, facial scars due to childhood accident, nose-mouth disfigurement). In these latter cases, we found no indications in the verbatim description of social fears that the social phobia is directly due to any of the reported or observed disabilities or handicaps. We also closely examined cases reporting social fears of 'eating and drinking while someone watches' with regard to the presence of eating disorders (see below). We found only four cases with DSM-IV eating disorders who reported fairly isolated fears of this type; a number small enough to have no important impact on estimated prevalence. 
Table 1. Lifetime cumulative incidence (LT-CI) and 12-month prevalence of generalized and non-generalized social phobia among 14-24 year-olds and relative persistence (ratio LT to 12-month rates)

\begin{tabular}{|c|c|c|c|c|c|c|c|c|c|c|c|c|c|c|c|c|c|c|c|c|}
\hline \multirow[b]{3}{*}{ Diagnostic group/age } & \multicolumn{9}{|c|}{ LT-CI } & \multicolumn{9}{|c|}{ 12-month prevalence } & \multirow{2}{*}{\multicolumn{2}{|c|}{$\begin{array}{l}\text { 12-m: LT ratio } \\
\text { persistence }\end{array}$}} \\
\hline & \multicolumn{3}{|c|}{ Male } & \multirow[b]{2}{*}{$P$} & \multicolumn{3}{|c|}{ Female } & \multicolumn{2}{|c|}{ Total } & \multicolumn{3}{|c|}{ Male } & \multirow[b]{2}{*}{$P$} & \multicolumn{3}{|c|}{ Female } & \multicolumn{2}{|c|}{ Total } & & \\
\hline & $N$ & $\%$ & S.E. & & $N$ & $\%$ & S.E. & $N$ & $\%$ & $N$ & $\%$ & S.E. & & $N$ & $\%$ & S.E. & $N$ & $\%$ & Male & Female \\
\hline \multicolumn{21}{|l|}{ Non-generalized SP } \\
\hline 14-17 years & 10 & $2 \cdot 2$ & 0.51 & & 17 & $3 \cdot 7$ & 0.72 & 27 & $2 \cdot 9$ & 7 & 1.5 & 0.71 & & 13 & $2 \cdot 9$ & 0.93 & 20 & $2 \cdot 2$ & $68 \cdot 2$ & 78.4 \\
\hline $18-24$ years & 45 & $4 \cdot 4$ & 0.87 & & 83 & 7.8 & 1.6 & 128 & $6 \cdot 1$ & 25 & $2 \cdot 4$ & $0.74 *$ & 0.001 & 56 & $5 \cdot 2$ & $0 \cdot 12$ & 81 & $3 \cdot 9$ & 54.5 & 66.7 \\
\hline Total & 55 & 3.7 & $0.57^{*}$ & $0 \cdot 000$ & 100 & 6.5 & 0.72 & 155 & $5 \cdot 12$ & 33 & $2 \cdot 2$ & $0.43 *$ & & 69 & $4 \cdot 5$ & 0.61 & 102 & $3 \cdot 4$ & $59 \cdot 5$ & $69 \cdot 2$ \\
\hline \multicolumn{21}{|l|}{ Generalized SP } \\
\hline $14-17$ years & 3 & $0 \cdot 6$ & 0.52 & & 8 & 1.8 & 0.52 & 11 & $1 \cdot 2$ & 2 & $0 \cdot 4$ & $0 \cdot 29$ & & 6 & $1 \cdot 3$ & $0 \cdot 82$ & 8 & $0 \cdot 9$ & $66 \cdot 7$ & $72 \cdot 2$ \\
\hline $18-24$ years & 16 & 1.6 & 0.59 & & 38 & 3.6 & 0.91 & 54 & $2 \cdot 6$ & 14 & 1.5 & 0.7 & & 35 & $3 \cdot 3$ & 0.91 & 49 & $2 \cdot 3$ & 93.7 & 91.7 \\
\hline Total & 19 & $1 \cdot 3$ & $0 \cdot 30^{*}$ & 0.001 & 46 & $3 \cdot 0$ & 0.52 & 65 & $2 \cdot 2$ & 16 & $1 \cdot 0$ & $0 \cdot 28^{*}$ & 0.001 & 41 & $2 \cdot 7$ & $0 \cdot 50$ & 57 & 1.9 & $76 \cdot 8$ & $90 \cdot 0$ \\
\hline \multicolumn{21}{|l|}{ DSM-IV social phobia } \\
\hline $14-17$ years & 13 & $2 \cdot 7$ & 0.78 & & 24 & $5 \cdot 5$ & 0.99 & 37 & $4 \cdot 0$ & 9 & $2 \cdot 0$ & 0.74 & & 19 & $4 \cdot 3$ & 0.93 & 28 & $3 \cdot 0$ & $74 \cdot 1$ & $78 \cdot 2$ \\
\hline $18-24$ years & 61 & $5 \cdot 9$ & 0.93 & & 121 & $11 \cdot 3$ & 1.5 & 182 & $8 \cdot 7$ & 39 & 3.8 & $0.95^{*}$ & 0.000 & 91 & $8 \cdot 5$ & $1 \cdot 3$ & 130 & $6 \cdot 2$ & $54 \cdot 4$ & $75 \cdot 2$ \\
\hline Total & 74 & $4 \cdot 9$ & $0.64 *$ & $0 \cdot 000$ & 146 & $9 \cdot 5$ & 0.87 & 220 & $7 \cdot 3$ & 48 & $3 \cdot 2$ & $0.51^{*}$ & 0.000 & 110 & $7 \cdot 2$ & 0.78 & 158 & $5 \cdot 2$ & $65 \cdot 3$ & $75 \cdot 8$ \\
\hline
\end{tabular}

Table 2. Lifetime $(L T)$ prevalence of social fears in the total sample and among those with DSM-IV generalized (G-SP) and nongeneralized (NG-SP) social phobia with social fear conditional probabilities for diagnosis

\begin{tabular}{|c|c|c|c|c|c|c|c|c|c|c|c|c|c|c|c|c|c|c|c|c|c|c|c|c|c|c|c|}
\hline \multirow[b]{3}{*}{ Social fear situation } & \multicolumn{7}{|c|}{ LT-prevalence in total sample } & \multicolumn{6}{|c|}{ Prevalence among NG-SP } & \multicolumn{7}{|c|}{ Prevalence among G-SP } & \multicolumn{7}{|c|}{ Cond. prob. for diagnosis } \\
\hline & \multicolumn{2}{|c|}{ Male } & \multirow[b]{2}{*}{$P$} & \multicolumn{2}{|c|}{ Female } & \multicolumn{2}{|c|}{ Total } & \multicolumn{2}{|c|}{ Male } & \multicolumn{2}{|c|}{ Female } & \multicolumn{2}{|c|}{ Total } & \multicolumn{2}{|c|}{ Male } & \multirow[b]{2}{*}{$P$} & \multicolumn{2}{|c|}{ Female } & \multicolumn{2}{|c|}{ Total } & \multicolumn{2}{|c|}{ Male } & \multirow[b]{2}{*}{$P$} & \multicolumn{2}{|c|}{ Female } & \multicolumn{2}{|c|}{ Total } \\
\hline & $N$ & $\%$ & & $N$ & $\%$ & $N$ & $\%$ & $N$ & $\%$ & $N$ & $\%$ & $N$ & $\%$ & $N$ & $\%$ & & $N$ & $\%$ & $N$ & $\%$ & $N$ & $\%$ & & $N$ & $\%$ & $N$ & $\%$ \\
\hline $\begin{array}{l}\text { Eating or drinking } \\
\text { in public }\end{array}$ & 36 & $2 \cdot 4^{*}$ & $0 \cdot 001$ & 98 & $6 \cdot 4$ & 134 & $4 \cdot 4$ & 6 & $11 \cdot 3$ & 7 & $7 \cdot 2$ & 13 & $8 \cdot 7$ & 5 & $27 \cdot 7$ & 0.049 & 25 & $54 \cdot 7$ & 30 & $46 \cdot 9$ & 11 & $31 \cdot 6$ & - & 32 & $33 \cdot 1$ & 44 & $32 \cdot 7$ \\
\hline $\begin{array}{l}\text { Writing while } \\
\text { someone watches }\end{array}$ & 25 & $1 \cdot 7 *$ & 0.04 & 42 & $2 \cdot 8$ & 67 & $2 \cdot 2$ & 0 & 0 & 4 & 3.8 & 4 & $2 \cdot 4$ & 4 & $22 \cdot 5$ & - & 10 & $21 \cdot 1$ & 14 & $21 \cdot 5$ & 4 & $17 \cdot 0$ & - & 14 & $32 \cdot 1$ & 18 & $26 \cdot 5$ \\
\hline $\begin{array}{l}\text { Participating in } \\
\text { social events }\end{array}$ & 44 & $3 \cdot 0^{*}$ & 0.001 & 95 & $6 \cdot 2$ & 139 & $4 \cdot 6$ & 4 & $7 \cdot 6$ & 5 & $4 \cdot 7$ & 9 & $5 \cdot 7$ & 13 & $70 \cdot 5$ & - & 30 & $64 \cdot 5$ & 43 & $66 \cdot 2$ & 17 & $39 \cdot 3$ & - & 34 & $36 \cdot 2$ & 52 & $37 \cdot 2$ \\
\hline $\begin{array}{l}\text { Performance/test } \\
\text { situations }\end{array}$ & 215 & $14 \cdot 4$ & 0.001 & 334 & $21 \cdot 9$ & 550 & $18 \cdot 2$ & 34 & $61 \cdot 6$ & 60 & $59 \cdot 9$ & 94 & $60 \cdot 5$ & 12 & $66 \cdot 0$ & 0.051 & 40 & 87.0 & 52 & 81 & 46 & $21 \cdot 5$ & 0.029 & 100 & $29 \cdot 9$ & 146 & $26 \cdot 6$ \\
\hline $\begin{array}{l}\text { Public speaking (in } \\
\text { front of others) }\end{array}$ & 159 & $10 \cdot 7$ & 0.001 & 239 & $15 \cdot 6$ & 398 & $13 \cdot 2$ & 25 & $45 \cdot 7$ & 46 & $45 \cdot 9$ & 71 & $45 \cdot 8$ & 14 & $75 \cdot 4$ & - & 42 & $91 \cdot 4$ & 56 & $86 \cdot 8$ & 39 & $24 \cdot 6$ & $0 \cdot 011$ & 88 & $36 \cdot 8$ & 127 & $31 \cdot 9$ \\
\hline $\begin{array}{l}\text { Talking with/to } \\
\text { others (social talk) }\end{array}$ & 63 & $4 \cdot 2^{*}$ & 0.001 & 131 & $8 \cdot 5$ & 194 & $6 \cdot 4$ & 9 & $15 \cdot 9$ & 12 & $12 \cdot 1$ & 21 & $13 \cdot 5$ & 15 & $78 \cdot 8$ & - & 35 & $75 \cdot 5$ & 49 & $76 \cdot 5$ & 23 & 37 & - & 47 & $35 \cdot 9$ & 70 & $36 \cdot 2$ \\
\hline Any social fear & 334 & $22 \cdot 3$ & 0.001 & 492 & $32 \cdot 2$ & 826 & $27 \cdot 3$ & - & - & - & - & - & - & - & - & - & - & - & - & - & - & - & - & - & - & - & - \\
\hline
\end{tabular}




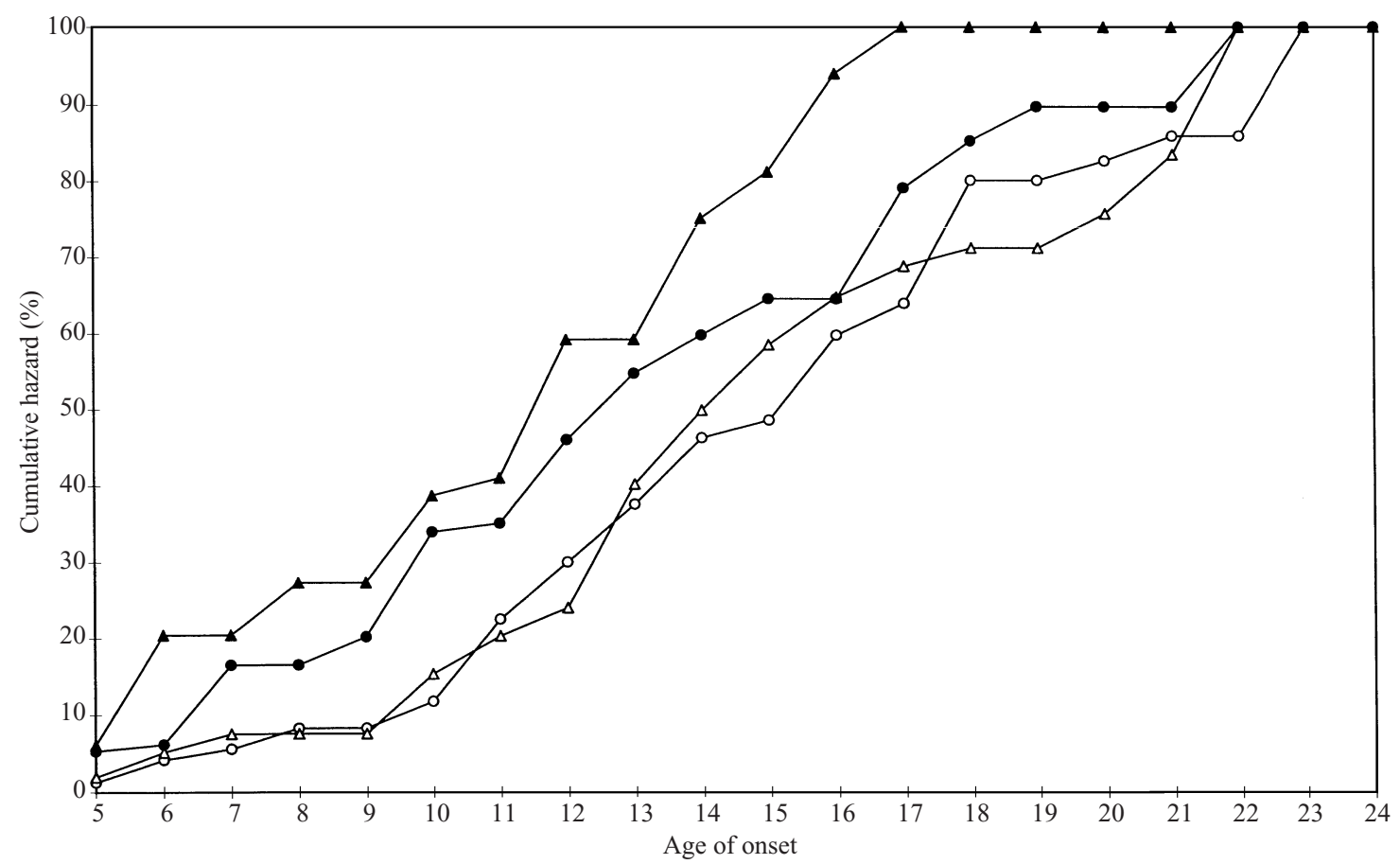

FIG. 1. Cumulative hazard function of generalized ( by gender.

\section{Social fears and social phobics}

Subjectively perceived unreasonably strong fears of doing things in front of other people or being in the centre of attention are much more widespread in our sample than social phobia as a diagnosis (Table 2). A persistent strong fear of this type at some time in their life, was acknowledged by $22.3 \%$ of male and $32.2 \%$ of female respondents. Women were significantly more likely to report these fears than men and were slightly more likely to report multiple fears than men $(P<0.04)$. The lifetime prevalence of specific social fears ranged from a low of $2 \cdot 1 \%$ for unreasonably strong fear of writing while someone watches, to a high of $18.2 \%$ for unreasonably strong fears of tests and examinations even though well prepared. Overall, the second most prevalent fear was public speaking $(13.2 \%)$ in front of a group of people in class or meetings.

Non-generalized social phobics are mainly characterized by fear of test/performance $(60.5 \%)$ and of speaking in front of a group $(45 \cdot 8 \%)$. Typical examples given by social phobics in this age group mainly to schoolrelated speaking situations. The only other noteworthy type of fear reported was talking to or with others. Examples given indicate that respondents with this type of fear referred mostly to situations that could be summarized best under the heading 'dealing with authorities'. Respondents with generalized social phobia differed markedly with regard to a higher frequency of almost all social fears as well as a more general nature of their verbatim examples. In an attempt to identify whether any specific social fear is associated particularly with social phobia, conditional probabilities (CP) of a DSM-IV diagnosis of social phobia given each of the six social fear clusters were calculated. We could not identify interpretable subtype-specific patterns of symptomatology. The only two noteworthy differences were: $(a)$ higher overall rates of symptoms; and $(b)$ higher rates of fear of humiliation among generalized social phobics. CPs were found to range from a low of $26.5 \%$ for fear of writing to a high of $37.2 \%$ for participating in social events. CPs of social phobia among those with a fear were found to 
Table 3. Lifetime association of social phobia with selected psychiatric disorders

\begin{tabular}{|c|c|c|c|c|c|c|c|c|c|c|c|c|}
\hline \multirow[b]{3}{*}{ Co-occurring disorders } & \multicolumn{4}{|c|}{ Any } & \multicolumn{4}{|c|}{ Non-generalized } & \multicolumn{4}{|c|}{ Generalized } \\
\hline & \multicolumn{2}{|c|}{$\begin{array}{l}\text { Row Dx \% by } \\
\text { phobia status }\end{array}$} & \multicolumn{2}{|c|}{ Univariate† } & \multicolumn{2}{|c|}{$\begin{array}{l}\text { Row Dx \% by } \\
\text { phobia status }\end{array}$} & \multicolumn{2}{|c|}{ Univariate† } & \multicolumn{2}{|c|}{$\begin{array}{l}\text { Row Dx \% by } \\
\text { phobia status }\end{array}$} & \multicolumn{2}{|c|}{ Univariate $\dagger$} \\
\hline & No & Yes & POR & $95 \% \mathrm{CI}$ & No & Yes & POR & $95 \% \mathrm{CI}$ & No & Yes & POR & $95 \%$ CI \\
\hline Any of the disorders below & $46 \cdot 1$ & $72 \cdot 1$ & $2 \cdot 79^{*}$ & $(1 \cdot 96-3 \cdot 98)$ & $46 \cdot 1$ & $65 \cdot 5$ & $2 \cdot 01^{*}$ & $(1 \cdot 35-2 \cdot 99)$ & $46 \cdot 1$ & 87.8 & $8 \cdot 26^{*}$ & $(3 \cdot 56-19 \cdot 18)$ \\
\hline Substance disorders & $27 \cdot 9$ & $41 \cdot 3$ & $1 \cdot 64^{*}$ & $(1 \cdot 17-2 \cdot 30)$ & $27 \cdot 9$ & $37 \cdot 6$ & $1 \cdot 39$ & $(0 \cdot 93-2 \cdot 07)$ & 27.9 & $50 \cdot 0$ & $2 \cdot 45^{*}$ & $(1 \cdot 37-4 \cdot 38)$ \\
\hline Nicotine & $17 \cdot 8$ & $31 \cdot 9$ & $2 \cdot 00^{*}$ & $(1 \cdot 40-2 \cdot 86)$ & $17 \cdot 8$ & $28 \cdot 7$ & $1 \cdot 70^{*}$ & $(1 \cdot 10-2 \cdot 61)$ & 17.8 & $39 \cdot 5$ & $2 \cdot 89^{*}$ & $(1 \cdot 58-5 \cdot 28)$ \\
\hline Alcohol ab/dep & $15 \cdot 6$ & $19 \cdot 4$ & $1 \cdot 14$ & $(0 \cdot 76-1 \cdot 72)$ & $15 \cdot 6$ & $19 \cdot 3$ & $1 \cdot 12$ & $(0 \cdot 69-1 \cdot 81)$ & 15.6 & $19 \cdot 5$ & $1 \cdot 19$ & $(0 \cdot 58-2 \cdot 46)$ \\
\hline Illicit drugs ab/dep & $4 \cdot 3$ & $7 \cdot 5$ & 1.67 & $(0 \cdot 89-3 \cdot 13)$ & $4 \cdot 3$ & 6.9 & $1 \cdot 52$ & $(0 \cdot 71-3 \cdot 26)$ & $4 \cdot 3$ & 8.9 & $2 \cdot 06$ & $(0.74-5.74)$ \\
\hline Any depressive disorders & $12 \cdot 1$ & $31 \cdot 1$ & $3 \cdot 04^{*}$ & $(2 \cdot 11-4 \cdot 37)$ & $12 \cdot 1$ & $25 \cdot 5$ & $2 \cdot 26^{*}$ & $(1 \cdot 45-3 \cdot 54)$ & $12 \cdot 1$ & $44 \cdot 4$ & $5 \cdot 58^{*}$ & $(3 \cdot 07-10 \cdot 14)$ \\
\hline Major depression & $10 \cdot 4$ & $25 \cdot 5$ & $2 \cdot 69^{*}$ & $(1 \cdot 82-3 \cdot 98)$ & $10 \cdot 4$ & $22 \cdot 5$ & $2 \cdot 25^{*}$ & $(1 \cdot 40-3 \cdot 61)$ & $10 \cdot 4$ & 32.5 & $3.95^{*}$ & $(2 \cdot 07-7 \cdot 51)$ \\
\hline Dysthymia & $2 \cdot 4$ & $10 \cdot 9$ & $5.03 *$ & $(2 \cdot 83-8 \cdot 95)$ & $2 \cdot 4$ & 3.7 & $1 \cdot 54$ & $(0.59-4.04)$ & $2 \cdot 4$ & 28.4 & $16 \cdot 29^{*}$ & $(8 \cdot 06-32 \cdot 95)$ \\
\hline Any anxiety disorders & $20 \cdot 8$ & $49 \cdot 9$ & $3 \cdot 71^{*}$ & $(2 \cdot 68-5 \cdot 13)$ & $20 \cdot 8$ & $43 \cdot 0$ & $2 \cdot 79 *$ & $(1 \cdot 90-4 \cdot 10)$ & $20 \cdot 8$ & $66 \cdot 5$ & $7 \cdot 44 * *$ & $(4 \cdot 16-13 \cdot 30)$ \\
\hline Panic disorder w/wo AG & $1 \cdot 3$ & $6 \cdot 2$ & $4 \cdot 68^{*}$ & $(2 \cdot 14-10 \cdot 27)$ & $1 \cdot 3$ & $4 \cdot 5$ & $3 \cdot 31^{*}$ & $(1 \cdot 22-9 \cdot 00)$ & $1 \cdot 3$ & $10 \cdot 1$ & $8 \cdot 32^{*}$ & $(2 \cdot 79-24 \cdot 82)$ \\
\hline Agoraphobia w/o PD & 1.8 & $8 \cdot 8$ & $5 \cdot 45^{*}$ & $(2 \cdot 87-10 \cdot 34)$ & 1.8 & $4 \cdot 3$ & $2 \cdot 45$ & $(0.92-6.52)$ & 1.8 & $19 \cdot 7$ & $13 \cdot 81^{*}$ & $(6 \cdot 30-30 \cdot 27)$ \\
\hline Specific phobias (+ NOS) & $17 \cdot 3$ & $43 \cdot 6$ & $3.67 *$ & $(2 \cdot 63-5 \cdot 10)$ & $17 \cdot 3$ & $38 \cdot 9$ & $3 \cdot 02 *$ & $(2 \cdot 04-4 \cdot 46)$ & $17 \cdot 3$ & $55 \cdot 0$ & $5 \cdot 80^{*}$ & $(3 \cdot 28-10 \cdot 25)$ \\
\hline GAD & 0.8 & $2 \cdot 3$ & $2 \cdot 42$ & $(0 \cdot 82-7 \cdot 11)$ & 0.8 & $0 \cdot 3$ & $0 \cdot 32$ & $(0.04-2.55)$ & 0.8 & 6.9 & $8 \cdot 10^{*}$ & $(2.53-25.90)$ \\
\hline OCD & 0.6 & $2 \cdot 3$ & $3.95^{*}$ & $(1 \cdot 25-12 \cdot 48)$ & 0.6 & $1 \cdot 3$ & $2 \cdot 03$ & $(0 \cdot 41-10 \cdot 04)$ & 0.6 & $4 \cdot 8$ & $8 \cdot 26^{*}$ & $(2 \cdot 13-31 \cdot 97)$ \\
\hline PTSD & $1 \cdot 0$ & $5 \cdot 9$ & $6 \cdot 23^{*}$ & $(2 \cdot 72-14 \cdot 26)$ & $1 \cdot 0$ & 0.7 & 0.69 & $(0 \cdot 09-5 \cdot 23)$ & $1 \cdot 0$ & $18 \cdot 5$ & $22 \cdot 65^{*}$ & $(9 \cdot 26-55 \cdot 40)$ \\
\hline Eating disorders & $2 \cdot 6$ & $5 \cdot 7$ & 1.99 & $(0 \cdot 93-4 \cdot 27)$ & $2 \cdot 6$ & $1 \cdot 8$ & $0 \cdot 60$ & $(0 \cdot 14-2 \cdot 60)$ & $2 \cdot 6$ & $15 \cdot 0$ & $6 \cdot 18^{*}$ & $(2 \cdot 54-15 \cdot 05)$ \\
\hline
\end{tabular}

Dx, diagnosis; POR, prevalence odds ratio; CI, confidence interval.

$\dagger$ Controls for age

$\chi_{1}^{2}, P<0.05$. 
Table 4. Current social role impairments, work productivity, help-seeking and treatment in 12-month pure and co-morbid generalized $(G-S P)$ and non-generalized (NG-SP) social phobia

\begin{tabular}{|c|c|c|c|c|c|c|c|c|c|c|c|c|c|c|c|c|c|c|c|c|c|c|}
\hline & \multicolumn{15}{|c|}{ 12-month DSM-IV social phobics } & \multirow{2}{*}{\multicolumn{7}{|c|}{ Total social phobia }} \\
\hline & \multicolumn{7}{|c|}{ NG-social phobia } & \multicolumn{8}{|c|}{ G-social phobia } & & & & & & & \\
\hline & & $\begin{array}{l}\text { ure } \\
=55 \text { ) }\end{array}$ & & $\begin{array}{r}\text { Co-1 } \\
(N\end{array}$ & $\begin{array}{l}\text { horbid } \\
=47)\end{array}$ & & $\begin{array}{l}\text { tal } \\
=102)\end{array}$ & & $\begin{array}{l}\text { Pure } \\
=11)\end{array}$ & & & $\begin{array}{l}\text { morbid } \\
I=46)\end{array}$ & & & $\begin{array}{l}\text { tal } \\
=57)\end{array}$ & & $\begin{array}{l}\text { Pure } \\
=66)\end{array}$ & \multirow[b]{2}{*}{$P$} & \multicolumn{2}{|c|}{$\begin{array}{l}\text { Co-morbid } \\
(N=93)\end{array}$} & \multicolumn{2}{|c|}{$\begin{array}{c}\text { Total } \\
(N=159)\end{array}$} \\
\hline & $N$ & $\%$ & $P$ & $N$ & $\%$ & $N$ & $\%$ & $N$ & $\%$ & $P$ & $N$ & $\%$ & $P$ & $N$ & $\%$ & $N$ & $\%$ & & $N$ & $\%$ & $N$ & $\%$ \\
\hline \multicolumn{23}{|l|}{$\begin{array}{l}\text { Current social role } \\
\text { impairments }\end{array}$} \\
\hline Work/school/household & 16 & $28 \cdot 4$ & - & 16 & $33 \cdot 9$ & 32 & $31 \cdot 4$ & 7 & $63 \cdot 6 \#$ & 0.028 & 25 & $55 \cdot 2 \#$ & 0.04 & 32 & $56 \cdot 7$ & 22 & $34 \cdot 1^{*}$ & $0 \cdot 190$ & 41 & $44 \cdot 4$ & 63 & $40 \cdot 2$ \\
\hline Leisure time activities & 3 & 5.0 & - & 15 & $31 \cdot 0$ & 18 & 17.6 & 1 & $10 \cdot 0$ & - & 16 & $35 \cdot 5$ & - & 17 & $30 \cdot 8$ & 4 & $5 \cdot 8^{*}$ & 0.001 & 31 & $33 \cdot 2$ & 35 & 21.9 \\
\hline $\begin{array}{l}\text { Social contacts/partner } \\
\text { relationship }\end{array}$ & 2 & $3 \cdot 1$ & - & 7 & $14 \cdot 6$ & 9 & 8.8 & 2 & $19 \cdot 5 \#$ & 0.037 & 18 & $39 \cdot 1$ \# & 0.008 & 20 & $35 \cdot 5$ & 4 & $5 \cdot 7^{*}$ & 0.001 & 25 & $26 \cdot 7$ & 29 & $18 \cdot 1$ \\
\hline \multicolumn{23}{|l|}{ Work days lost/productivity } \\
\hline Missed > 2 days/mo & 0 & 0 & - & 0 & 0 & 0 & 0 & 0 & 0 & - & 9 & $18 \cdot 7 \#$ & 0.002 & 9 & $15 \cdot 2$ & 0 & 0 & - & 9 & $9 \cdot 2$ & 9 & $5 \cdot 4$ \\
\hline $\begin{array}{l}\text { Diminished work } \\
\text { productivity }>2\end{array}$ & 9 & $15 \cdot 8$ & - & 16 & $34 \cdot 3$ & 25 & $24 \cdot 3$ & 0 & $0^{*}$ & 0.012 & 20 & $43 \cdot 2$ & - & 20 & $35 \cdot 2$ & 9 & $13 \cdot 2^{*}$ & 0.001 & 36 & $38 \cdot 7$ & 45 & $28 \cdot 2$ \\
\hline \multicolumn{23}{|l|}{$\begin{array}{l}\text { Help-seeking because of social } \\
\text { phobia }\end{array}$} \\
\hline Any medical doctor & 3 & $5 \cdot 6$ & - & 2 & $4 \cdot 3$ & 5 & $5 \cdot 0$ & 1 & $10 \cdot 0$ & - & 14 & $30 \cdot 4 \#$ & $0 \cdot 001$ & 15 & $26 \cdot 8$ & 5 & $7 \cdot 7$ & - & 16 & $17 \cdot 2$ & 21 & $13 \cdot 3$ \\
\hline Any other MH-professional & 2 & $3 \cdot 6$ & - & 5 & $10 \cdot 6$ & 7 & 6.9 & 1 & $10 \cdot 0$ & - & 6 & $13 \cdot 0$ & - & 7 & $12 \cdot 5$ & 3 & $4 \cdot 6$ & - & 11 & $11 \cdot 8$ & 14 & 8.9 \\
\hline Total any help-seeking & 6 & $10 \cdot 9$ & - & 7 & $14 \cdot 9$ & 13 & $12 \cdot 7$ & 3 & $27 \cdot 3$ & - & 20 & $43 \cdot 5 \#$ & 0.002 & 23 & $40 \cdot 4$ & 8 & $12 \cdot 3 *$ & 0.019 & 26 & $28 \cdot 0$ & 34 & $21 \cdot 5$ \\
\hline
\end{tabular}

* $P<0.05$ pure $v$. co-morbid $\left(\chi^{2}\right)$.

P $P<0.05$ pure $v$. co-morbid $\left(\chi^{2}\right)$.
\# $P<0.05$ generalized versus non-generalized social phobia $\left(\chi^{2}\right)$. 
be significantly greater for females than males. It is noteworthy that the $\mathrm{CP}$ of social phobia increases steadily with number of social fears reported, from a low of $22.4 \%$ for those with just one social fear to a high of $62 \cdot 2 \%$ for those with five or six fears.

\section{Age of first onset of social phobia}

Kaplan-Meier cumulative age of onset curves based on retrospective reports were computed separately for males and females. As shown in Fig. 1, the curves between non-generalized and generalized types differ significantly (log rank: 4,93 , df $=1, P=0 \cdot 0265$ ). Most social phobias among respondents in the age range of the sample start between ages 10 and 21. But the generalized type has a significantly lower median age of onset (11.5 v. 14 for males, $P<0.004$ and $12.5 v .15$ for females, $P<0.003)$ than nongeneralized social phobia.

\section{Co-morbidity}

Table 3 presents data on co-morbidities of lifetime social phobia with other DSM-IV/CIDI disorders. When controlling for gender and birth cohort only a few significant $(P<0.05)$ and relatively modest associations were found between non-generalized social phobia and the other disorders considered here. Co-morbidities are much stronger and consistent for generalized social phobia, especially with regard to specific phobia $(\mathrm{OR}=16 \cdot 4)$, post-traumatic stress disorder $(\mathrm{OR}=17 \cdot 3)$, and dysthymia $(\mathrm{OR}=13 \cdot 7)$. A more detailed analysis revealed that taking a time difference of at least 1 year, social phobia precedes $85.2 \%$ of the co-morbid substance use disorders, $81 \cdot 6 \%$ of the depressive disorders and $64.4 \%$ of the other anxiety disorders, with specific phobia being the only co-morbid condition for which earlier ages of onset were reported relatively frequently.

The significant associations of social phobia with eating disorder and panic disorder deserve attention because of the possibility that in the first case social phobia might occur only as part of the characteristic symptom cluster of eating disorders and in the second case that social phobia might be part of panic-related avoidance behaviour. A case-by-case review of all eating disorders revealed that this assumption is rather unlikely because of the following reasons: $(a)$ all but two of these cases have several social fears, not just fears of eating and drinking with others; (b) the onset of social fears occurred in most cases before the onset of the eating disorders; and $(c)$ only few cases with any eating disorder had a full-blown CIDI-DSM-IV diagnosis 'anorexia nervosa' or 'bulimia', but rather binge eating or subthreshold eating disorder (NOS). For panic disorder we found that although social fears are very frequent, the separate diagnosis of panic disorder is justified in all cases, because of: (a) the consistently earlier age of onset of social phobia than panic disorder; $(b)$ the panic disorder cases' report that panic attacks only sometimes occur in social fear situations; and (c) the primary cognitions of panic disorder cases of being afraid of getting another unexpected attack, having to die, or to get a heart attack, that are not reported by social phobics in the M-CIDI social phobia section.

\section{Impairments, help-seeking and treatment}

The upper part of Table 4 presents data on current social phobia-specific role impairments among those with diagnoses of pure and comorbid 12-month social phobia. Impairments in work, school or household management, but not with regard to leisure time activities, are more frequent in pure $(P=0.028)$ and comorbid $(P=0.04)$ generalized than nongeneralized social phobics. The presence of comorbid conditions is associated consistently with increased social-role impairments. About onefifth of co-morbid generalized social phobics indicated having been unable to go to school or work for more than 2 days in the past month because of their social phobia. In addition, a substantial proportion of co-morbid generalized $(43.2 \%)$ and non-generalized $(34.3 \%)$ social phobics reported significantly diminished work productivity.

The lower part of Table 4 reports findings about professional help-seeking and treatment. Smaller proportions of those with pure $(10.9 \%)$ and co-morbid (14.9\%) non-generalized social phobia than those with pure generalized $(27 \cdot 3 \%)$ or co-morbid generalized (43.5\%) social phobia contacted a MD or another mental health specialist (in the vast majority subjects indicated a psychologist) specifically because of their social phobia at some time in their life. It should be 
Table 5. Lifetime association of social phobia with sociodemographic and developmental factors

\begin{tabular}{|c|c|c|c|c|c|c|}
\hline & \multicolumn{2}{|c|}{ Any social phobia } & \multicolumn{2}{|c|}{ Non-generalized } & \multicolumn{2}{|c|}{ Generalized } \\
\hline & POR & $95 \% \mathrm{CI}$ & POR & $95 \% \mathrm{CI}$ & POR & $95 \% \mathrm{CI}$ \\
\hline \multicolumn{7}{|l|}{ Type of education $\dagger$} \\
\hline Hauptschule & $1 \cdot 26$ & $(0 \cdot 75-2 \cdot 10)$ & 1.09 & $(0 \cdot 59-2 \cdot 02)$ & $1 \cdot 72$ & $(0 \cdot 71-4 \cdot 16)$ \\
\hline Fachhochschule & $1 \cdot 35$ & $(0 \cdot 69-2 \cdot 65)$ & $1 \cdot 35$ & $(0 \cdot 63-2 \cdot 92)$ & $1 \cdot 37$ & $(0 \cdot 38-4 \cdot 94)$ \\
\hline Realschule & $1 \cdot 40$ & $(0 \cdot 92-2 \cdot 13)$ & $1 \cdot 33$ & $(0 \cdot 81-2 \cdot 16)$ & $1 \cdot 59$ & $(0 \cdot 74-3 \cdot 41)$ \\
\hline Gymnasium & & 1.00 & & $1 \cdot 00$ & & 1.00 \\
\hline University & $1 \cdot 18$ & $(0 \cdot 80-1 \cdot 74)$ & $1 \cdot 13$ & $(0 \cdot 72-1 \cdot 77)$ & $1 \cdot 33$ & $(0 \cdot 65-2 \cdot 72)$ \\
\hline \multicolumn{7}{|l|}{ Living arrangement } \\
\hline With parents & $1 \cdot 00$ & & 1.00 & & $1 \cdot 00$ & \\
\hline Alone & $1 \cdot 40$ & $(0.99-1.96)$ & $1 \cdot 29$ & $(0.84-1 \cdot 96)$ & $1 \cdot 59$ & $(0 \cdot 92-2 \cdot 77)$ \\
\hline With partner & $1 \cdot 38$ & $(0 \cdot 88-2 \cdot 17)$ & $1.94^{*}$ & $(1 \cdot 19-3 \cdot 17)$ & $0 \cdot 31$ & $(0 \cdot 08-1 \cdot 20)$ \\
\hline With spouse & $1 \cdot 02$ & $(0 \cdot 51-2 \cdot 05)$ & $1 \cdot 58$ & $(0 \cdot 77-3 \cdot 25)$ & - & \\
\hline \multicolumn{7}{|l|}{ Subjective financial situation $\dagger$} \\
\hline Bad & $1 \cdot 67 *$ & $(1 \cdot 06-2 \cdot 64)$ & $1 \cdot 48$ & $(0 \cdot 85-2 \cdot 57)$ & $2 \cdot 12$ & $0 \cdot 99-4 \cdot 54)$ \\
\hline $\mathrm{OK}$ & $1 \cdot 36$ & $(0 \cdot 99-1 \cdot 86)$ & $1 \cdot 25$ & $(0 \cdot 86-1 \cdot 82)$ & $1 \cdot 65$ & $(0 \cdot 95-2 \cdot 85)$ \\
\hline Good & & 1.00 & & 1.00 & & 1.00 \\
\hline Very good & $0 \cdot 93$ & $(0 \cdot 56-1 \cdot 55)$ & $1 \cdot 16$ & $(0 \cdot 68-2 \cdot 00)$ & $0 \cdot 35$ & $(0 \cdot 08-1 \cdot 44)$ \\
\hline \multicolumn{7}{|l|}{ Urbanicity $\dagger$} \\
\hline Suburbs & $1 \cdot 00$ & & $1 \cdot 00$ & & $1 \cdot 00$ & \\
\hline Urban Munich & $1 \cdot 06$ & $(0 \cdot 76-1 \cdot 47)$ & $0 \cdot 98$ & $(0 \cdot 67-1 \cdot 43)$ & $1 \cdot 30$ & $(0 \cdot 69-2 \cdot 42)$ \\
\hline \multicolumn{7}{|l|}{ Developmental risk factors } \\
\hline Behavioural inhibition $(z>0 \cdot 49)$ & $4 \cdot 54^{*}$ & $(3 \cdot 38-6 \cdot 09)$ & $2 \cdot 92^{*}$ & $(2 \cdot 10-4 \cdot 06)$ & $23 \cdot 73^{*}$ & $(10 \cdot 12-56 \cdot 68)$ \\
\hline Separated from parent & $2 \cdot 13^{*}$ & $(1 \cdot 57-2 \cdot 89)$ & $1 \cdot 79 *$ & $(1 \cdot 24-2 \cdot 59)$ & $3 \cdot 06^{*}$ & $(1 \cdot 83-5 \cdot 11)$ \\
\hline Parental separation & $1 \cdot 82 *$ & $(1 \cdot 36-2 \cdot 43)$ & $1 \cdot 60^{*}$ & $(1 \cdot 13-2 \cdot 26)$ & $2 \cdot 42 *$ & $(1 \cdot 47-4 \cdot 00)$ \\
\hline \multicolumn{7}{|l|}{ Parental history } \\
\hline Mental problems & $2 \cdot 01^{*}$ & $(1 \cdot 51-2 \cdot 68)$ & $1 \cdot 80^{*}$ & $(1 \cdot 29-2 \cdot 50)$ & $2 \cdot 68 *$ & $(1 \cdot 57-4 \cdot 58)$ \\
\hline Treatment & $1 \cdot 84 *$ & $(1 \cdot 28-2 \cdot 64)$ & $1.99 *$ & $(1 \cdot 32-3 \cdot 00)$ & $1 \cdot 52$ & $(0 \cdot 78-2 \cdot 97)$ \\
\hline Problems & $2 \cdot 19^{*}$ & $(1 \cdot 65-3 \cdot 91)$ & $1.92 *$ & $(1 \cdot 38-2 \cdot 66)$ & $3 \cdot 08 *$ & $(1 \cdot 81-5 \cdot 26)$ \\
\hline Substance problems & $1 \cdot 65^{*}$ & $(1 \cdot 21-2 \cdot 24)$ & $1 \cdot 29$ & $(0 \cdot 88-1 \cdot 88)$ & $2 \cdot 73^{*}$ & $(1 \cdot 65-4 \cdot 54)$ \\
\hline Treatment & $1.83 *$ & $(1 \cdot 17-2 \cdot 85)$ & $1 \cdot 45$ & $(0 \cdot 83-2 \cdot 56)$ & $2 \cdot 81 *$ & $(1 \cdot 43-5 \cdot 50)$ \\
\hline Problems & $1 \cdot 73^{*}$ & $(1 \cdot 27-2 \cdot 35)$ & $1 \cdot 35$ & $(0.93-1.97)$ & $2 \cdot 87^{*}$ & $(1 \cdot 73-4 \cdot 76)$ \\
\hline
\end{tabular}

Dx, diagnosis; POR, prevalence odds ratio; CI, confidence interval.

$\dagger$ Controls for gender and birth-cohort.

* $\chi_{1}^{2}, P<0.05$.

noted that these findings do not necessarily mean that adequate treatment was actually provided.

\section{Risk factors and correlates of non-generalized and generalized social phobia}

When controlling for age and gender, only a few psychosocial correlates of social phobia were identified in Table 5. Although we examined several other variables ranging from social class to social network variables, only poor financial situation $(\mathrm{OR}=1.67)$ and, surprisingly, living with a partner (1.94) revealed significant associations with non-generalized social phobia. Developmental variables were more strongly related to generalized social phobia, including retrospectively assessed high 'behavioural inhibition' between ages 5 to 12, 'long-lasting separation from either parent during childhood or early adolescence' and 'parental history of psychopathology'. The bivariate associations suggest, that all of these variables, particularly behavioural inhibition, are much more strongly related to the onset of generalized social phobia than non-generalized social phobia.

\section{DISCUSSION}

This community study reporting data on the prevalence, clinical characteristics, impairments, risk factors and co-morbidity patterns of DSMIV social phobia in 3021 respondents aged 14-24 confirms that social phobia is a quite prevalent disorder in this age group. In comparison with previous epidemiological studies with considerably broader age groups (Schneier et al. 1992; Davidson et al. 1993; Degonda \& Angst, 1993; Magee et al. 1996), the focus on a sample of 
adolescents and young adults allows a more detailed description of early stages of this disorder, before more severe complications are present (Hazen \& Stein, 1995). We find that DSM-IV social phobia, with a lifetime rate of $9.5 \%$ for females and $4.9 \%$ for males, is considerably more prevalent than in the early DSM-III/DIS studies but not as prevalent as in the more recent DSM-III-R/CIDI studies (ranging from 13.3 to $16 \%$ ) that have been based on samples with an age range, extending throughout adulthood. Our higher prevalence than in the DIS studies is probably due to a more complete assessment of qualifying social fears and especially the younger age groups in the current study, whereas the lower rates as compared to the NCS (Magee et al. 1996), which used DSM-III-R, might be due to the use of stricter impairment and distress criteria as well as a more refined symptom assessment in DSMIV. Although these prevalence were estimated without taking into account the diagnostic exclusion criteria for DSM-IV, a closer inspection revealed that in this young age group, none of the DSM-IV axis I diagnostic exclusionary considerations examined (substances, somatic conditions, mental disorders) plays a critical role. As a limitation, however, one should acknowledge that our study does not allow to investigate the relationship to avoidant and other personality disorders.

Although there is still considerable controversy about whether generalized social phobia is a distinct clinical syndrome and even more disagreement about its diagnostic assessment (Heimberg et al. 1990, 1993; Manuzza et al. 1995), our findings provide epidemiological support for the usefulness of the DSM-IV subtype of generalized social phobia. 'Generalized social phobia' as defined in this paper simply by number of trigger situations, differs markedly from non-generalized social phobia not only in symptomatology, age of onset patterns, co-morbidity, impairment, and treatment rates, but also with regard to the degree of associations with several family and developmental variables, such as retrospectively assessed parental psychopathology, early separation from either parent and childhood inhibition. Non-generalized social phobics in this age group are described best as respondents experiencing fear reactions and avoidance in test and performance situations in school or other educational settings or less frequently in dealing with authorities. The generalized subtype is not only characterized by: $(a)$ social fears in many or even all types of situational clusters assessed: and $(b)$ by a higher number of associated symptoms, such as fear of humiliation, paniclike symptoms and extreme distress: but $(c)$ as judged from the verbatim examples - also reveals a more generalized fear and avoidance pattern in everyday interactional situations. Most frequent examples were persistent everyday social fear situations like "talking to others, asking for directions, initiating contacts, participating in any type of social event and being fearful in any situation where he or she might be in the center of attention of others'. These more general interactional fears, characteristic of generalized social phobia, were found to start considerably earlier in life than test and performance as well as public speaking fears characteristic for non-generalized social phobia. The cumulative age of onset curve suggests that $50 \%$ of the generalized, but only $19 \%$ of the non-generalized, social phobics started before the age of 12. In light of the early onset of generalized social phobia, it should be noted as a limitation that this study made no attempt to assess the role of particular types of personality disorders and did not explore the role of other potentially important subtypes of social phobia considered by the APA-DSM-IV Task Force (Heimberg et al. 1993). It also should be noted that the definition of generalized phobia is limited by the number and the specific types of situations included in the M-CIDI.

Consistent with many other studies (Schneier et al. 1992; Magee et al. 1996), social phobia was found to be highly co-morbid with other mental disorders, particularly other anxiety disorders and depressive disorders. But it is important to note that the diagnostic overlap is mainly driven by the generalized subtype. For non-generalized social phobias only very few significant, rather moderate odds ratios were found. In contrast, more than two-thirds of generalized social phobics reported a history of at least one other M-CIDI disorder, with highest odds-ratios for anxiety disorders and dysthymia (13.7), moderate odds-ratios for eating disorder (4.9), major depressive episodes (3.5) and nicotine dependence (2.9), but not other types of substances use 
disorders. Given that co-morbidity rates increased steadily by age group, and that the vast majority of these co-morbid conditions, except for simple phobia, had a considerably later age of onset than social phobia, we can assume that generalized social phobia among adolescents and young adults is a risk factor for the subsequent development of other mental disorders. Contrary to the findings in studies in adults, however, we could not yet find high comorbidity with alcohol use disorders (Schneier et al. 1992; Magee et al. 1996) or with secondary major depressive episodes (Van Ameringen et al. 1991).

The impairment findings are especially striking in that they suggest that generalized social phobia can be quite impairing even in fairly young age groups, especially with regard to school and work performance. This result is consistent with other recent research on the impact of chronic social phobia in adults (Davidson et al. 1993, 1994; Wittchen \& Beloch, 1996; Kessler et al. 1999) and shows that this condition is, in fact, a very important one from a health economic and public health perspective. It is also essential to note that this is true also for pure generalized social phobia, although the presence of co-morbid conditions increases substantially the degree and the frequency of impairments and disability related to social phobia. Non-generalized social phobia - at least in 14- to 24-year-olds - however, is less persistent and less frequently associated with considerable impairments, unless complicated by co-morbidity.

We also confirm previous findings (Magee et al. 1996) of overall low treatment utilization, with approximately only one out of four lifetime social phobics ever having received some kind of mental health treatment for this condition. This finding was unexpected in light of the fact that the German health care system offers almost everybody free access to a wide range of mental health care free of charge. Nevertheless, our rates for social phobia-specific help-seeking and treatment rates are only slightly higher than estimates from the US. Magee et al. 1996) reported for the NCS professional help-seeking rates with identical questions of $19 \%$ as compared to $22 \%$ in the present study.

Concerning risk factors for onset, it is noteworthy that aside from the effect from gender, poor financial situation and living with a partner, we could not replicate earlier positive associations of poor educational status, never been married, being unemployed or homemaker and living with parents with social phobia (Magee et al. 1996). This failure might be explained with the fairly young age group used in our study and suggests that these variables are rather consequences of the long-term course than risk factors for the onset of social phobia. In agreement with some genetic and family genetic studies suggesting a moderate heritability of social phobia and a moderate threefold increased relative risk (Knowles et al. 1995; Stein et al. 1998), we find that respondents reporting a positive family history of mental problems, particularly anxiety and depressive disorders, are significantly more likely to have generalized social phobia. Also, other retrospectively reported developmental predictors were significant, such as early separation from either parent and, most impressively, behavioural inhibition. Our findings concerning the childhood temperament construct of behavioural inhibition to the unfamiliar is in agreement with hypotheses (Kagan, 1989) as well as the work of Biederman et al. (1990) suggesting that behavioural inhibition might be an important construct for describing early individual vulnerabilities and risks for the onset of generalized and possibly non-generalized social phobia.

This work is part of the Early Developmental Stages of Psychopathology (EDSP) study and is funded by the German Federal Ministry for Research and Technology, project no. $01 \mathrm{~EB} 9405 / 6$ and it is also supported by an unrestricted educational grant from SmithKline Beecham Pharmaceuticals.

The EDSP is a 5 year prospective epidemiological study funded by the German Federal Ministry of Research and Technology to investigate the prevalence of substance use and other mental disorders, their early development and progression, and patterns of co-morbidity. H.-U. Wittchen is the principal investigator. Scientific advisors are Dr J. Angst (Zurich), Dr W. Esser (Mannheim), Dr K. Merikangas (New Haven) and Dr R. C. Kessler (Boston). Collaborating sites and investigators are Dr G. Bhhringer (Institut für Therapieforschung, München) and Dr U. John (Medizinische Hochschule, Lübeck). Staff members of the EDSP group are: E. Beloch, E. Garczynski, A. Holly, A. Perkonigg, H. 
Pfister, V. Reed, P. Schuster, D. Türk, A. Vossen, and U. Wunderlich.

An English version of the M-CIDI interview used in this study, including the lifetime and 12-months version with respondents booklets and manuals, as well as the diagnostic programmes (Wittchen \& Pfister, 1997) are available, in English, by writing to H.-U. Wittchen.

\section{REFERENCES}

Allgulander, C. (1989). Psychoactive drug use in a general population sample, Sweden: correlates with perceived health, psychiatric diagnoses, and mortality in an automated record-linkage study. American Journal of Public Health 79, 1006-1010.

Angst, J. \& Dobler-Mikola, A. (1985). The Zurich study - V. Anxiety and phobia in young adults. European Archives of Psychiatric Neurological Science 234, 408-418.

Biederman, J., Rosenbaum, J. F., Hirshfeld, D. R., Farone, S. V., Bolduc, E. A., Gersten, M., Meminger, S. R., Kagan, J., Snidman, N. \& Reznick, J. S. (1990). Psychiatric correlates of behavioral inhibition in young children of parents with and without psychiatric disorders. Archives of General Psychiatry 47, 21-26.

Bland, R. C., Orn, H. \& Newman, S. C. (1988). Lifetime prevalence of psychiatric disorders in Edmonton. Acta Psychiatrica Scandinavica 338, 24-32.

Bourdon, K. H. (1993). Social phobia: an overview of treatment strategies. Journal of Clinical Psychiatry 54, 165-171.

Bourdon, K. H., Boyd, J. H., Rae, D. S., Burns, B. J., Thompson,

J. W. \& Locke, B. Z. (1988). Gender differences in phobias: results of the ECA community survey. Journal of Anxiety Disorders 2 , $227-241$.

Bryant, B. \& Towers, P. E. (1974). Social difficulty in a student sample. British Journal of Educational Psychology 44, 13-21.

Burns, L. E. (1980). The epidemiology of fears and phobias in general practice. Journal of International Medical Research 8 (Suppl. 3), $1-8$.

Davidson, J. R. T., Hughes, D. L., George, L. K. \& Blazer, D. G. (1993). The epidemiology of social phobia: findings from the Duke Epidemiologic Catchment Area Study. Psychological Medicine 23, 709-718.

Davidson, J. R., Hughes, D. C., George, L. K. \& Blazer, D. G. (1994). The boundary of social phobia. Exploring the threshold. Archives of General Psychiatry 51, 975-983.

Degonda, M. \& Angst, J. (1993). The Zurich study, XX. Social phobia and agoraphobia. European Archives of Psychiatry and Clinical Neuroscience 243, 95-102.

DiNardo, P. A., O’Brien, G. T., Barlow, D. H., Waddell, M. T. \& Blanchard, E. B. (1983). Reliability of DSM-III anxiety disorder categories using a new structured interview. Archives of General Psychiatry 40, 1070-1074.

Hazen, A. L. \& Stein, M. (1995). Clinical phenomenology and comorbidity. In Social Phobia, Clinical and Research Perspectives (ed. M. B. Stein), pp. 3-41. American Psychiatric Press: Washington, DC.

Heimberg, R. G., Hope, D. A., Dodge, C. S. \& Becker, R. E. (1990). DSM-III-R subtypes of social phobia. Comparison of generalized social phobics and public speaking phobics. Journal of Nervous and Mental Disease 178, 172-179.

Heimberg, R. G., Hope, D. A. \& Dodge, C. S. (1993). DSM-III-R subtypes of social phobia: comparison of generalized social phobics and public speaking phobics. Journal of Anxiety Disorders 7, 249-269.

Kagan, J. (1989). Temperamental contributions to social behavior. American Psychology 44, 668-674.

Kessler, R. C., Stang, P., Wittchen, H.-U., Stein, M. \& Walters, E. E. (1999). Lifetime co-morbidities between social phobia and mood disorders in the US National Comorbidity Survey. Psychological Medicine (in the press).
Knowles, J. A., Mannuzza, S. \& Fyer, A. (1995). Heritability of social anxiety. In Social Phobia. Clinical and Research Perspectives (ed. M. B. Stein), pp. 147-162. Psychiatric Press: Washington, DC.

Liebowitz, M. R., Gorman, J. M., Fyer, A. J. \& Klein, D. F. (1985). Social phobia: review of a neglected anxiety disorder. Archives of General Psychiatry 42, 729-736.

Magee, W. J., Eaton, W. W., Wittchen, H.-U., McGonagle, K. A. \& Kessler, R. C. (1996). Agoraphobia, simple phobia, and social phobia in the National Comorbidity Survey. Archives of General Psychiatry 53, 159-168.

Mannuzza, S., Schneier, F. R., Chapman, T. F., Liebowitz, M. R., Klein, D. F. \& Fyer, A. J. (1995). Generalized social phobia. Reliability and validity. Archives of General Psychiatry 52, 230-237.

Marks, I. (1979). The classification of phobic disorders. British Journal of Psychiatry 116, 377-386.

Merikangas, K., Angst, J., Eaton, W., Canino, G., Rubio-Stipec, M., Wacker, H., Wittchen, H.-U., Andrade, L., Essau, C. A., Kraemer, H., Robins, L. \& Kupfer, D. (1996). Comorbidity and boundaries of affective disorders with anxiety disorders and substance abuse: results of an international task force. British Journal of Psychiatry 168 (suppl. 30), 49-58.

Mullaney, J. A. \& Trippet, C. J. (1979). Alcohol dependence and phobias: clinical description and relevance. British Journal of Psychiatry 135, 565-573.

Pollard, C. A. \& Henderson, J. G. (1988). Four types of social phobia in a community sample. Journal of Nervous and Mental Disease 176, $440-445$.

Reed, V., Gander, F., Pfister, H., Steiger, A., Sonntag, H., Trenkwalder, C., Hundt, W. \& Wittchen, H.-U. (1998). To what degree the CIDI correctly identifies DSM-IV disorders? Testing validity issues in a clinical sample. International Journal of Methods in Psychiatric Research 7, 142-155.

Regier, D. A., Narrow, W. E. \& Rae, D. S. (1990). The epidemiology of anxiety disorders: the Epidemiologic Catchment Area (ECA) experience. Journal of Psychiatric Research 24 (suppl. 2), 3-14.

Rezwick, J. S., Hegeman, I. M., Kaufman, E. R., Woods, S. W. \& Jacobs, M. (1992). Retrospective and concurrent self-report of behavioral inhibition and their relation to adult mental health. Development and Psychopathology 4, 301-321.

Robins, L. N., Helzer, J. E., Croughan, J. \& Ratcliff, K. S. (1981). National Institute of Mental Health Diagnostic Interview Schedule: its history, characteristic and validity. Archives of General Psychiatry 38, 381-389.

Rosenbaum, J. F., Biederman, J., Bolduc, E. A., Hirshfeld, D. R., Faraone, S. V. \& Kagan, J. (1992). Comorbidity of parental anxiety disorders as risk for childhood-onset anxiety in inhibited children. American Journal of Psychiatry 149, 475-481.

Ross, J. (1993). Social phobia: the consumer's perspective. Journal of Clinical Psychiatry 54 suppl. 5-9.

SAS (1996). SAS release 6.12. TS0020. SAS Institute: Cary, NC.

Schneier, F. R., Johnson, J., Hornig, C. D., Liebowitz, M. R. \& Weissman, M. M. (1992). Social phobia. Comorbidity and morbidity in an epidemiologic sample. Archives of General Psychiatry 49, 282-288.

Shah, B. V., Barnewell, B. G. \& Bieler, G. S. (1996). SUDAAN Release 7.11. Research Triangle Park: Cary, NC.

Stein, M. B., Walker, J. R. \& Forde, D. R. (1994). Setting diagnostic thresholds for social phobia: considerations from a community survey of social anxiety. American Journal of Psychiatry 151, $408-412$.

Stein, M. B., Walker, J. R. \& Forde, D. R. (1996). Public-speaking fears in a community sample. Prevalence, impact on functioning, and diagnostic classification. Archives of General Psychiatry 53, 169-174.

Stein, M. B., Chartier, M. J., Hazen, A. L., Kozak, M. V., Tancer, M. E., Lander, R. N., Furer, P., Chubaty, D. \& Walker, J. R. (1998). A direct-interview family study of generalized social phobia. American Journal of Psychiatry 155, 90-96.

Van Ameringen, M., Manicini, C. \& Styan, G. (1991). Relationship 
of social phobia with other psychiatric illness. Journal of Affective Disorders 21, 93-99.

Wacker, H. R., Mhllejans, R., Klein, K. H. \& Battegay, R. (1992). Identification of cases of anxiety disorders and affective disorders in the community according to ICD-10 and DSM-III-R using the Composite International Diagnostic Interview (CIDI). International Journal of Methods in Psychiatric Research 2, 91-100.

Walker, J. R. \& Stein, M. B. (1995). Epidemiology. In Social Phobia Clinical and Research Perspectives (ed. M. B. Stein), pp. 43-75. American Psychiatric Press: Washington, DC.

Wells, J. E., Bushnell, J. A., Hornblow, A. R., Joyce, P. R. \& Oakley-Browne, M. A. (1989). Christchurch psychiatric epidemiology study. I. Methodology and lifetime prevalence for specific psychiatric disorders. Australian and New Zealand Journal of Psychiatry 23, 315-326.

Wittchen, H.-U. (1994). Reliability and validity studies of the WHOComposite International Diagnostic Interview (CIDI): a critical review. Journal of Psychiatric Research 28, 57-84.

Wittchen, H.-U. (1996). Critical issues in the evaluation of comorbidity. British Journal of Psychiatry 168 (suppl. 30), 9-16.

Wittchen, H.-U. \& Beloch, E. (1996). The impact of social phobia on quality of life. International Clinical Psychopharmacology 11 (suppl. 3), 15-23.

Wittchen, H.-U. \& Pfister, H. (eds.). (1997). DIA-X-Interviews:
Manual für Screening-Verfahren und Interview; Interviewheft Längsschnittuntersuchung (DIA-X-Lifetime); Ergänzungsheft (DIA-X-Lifetime); Interviewheft Querschnittuntersuchung (DIAX-12 Monate); Erg@nzungsheft (DIA-X-12Monate); PCProgramm zur Durchfhhrung des Interviews (L@ngs- und Querschnittuntersuchung); Auswertungsprogramm. Frankfurt: Swets \& Zeitlinger.

Wittchen, H.-U., Essau, C. A., Zerssen, D. von, Krieg, C. J. \& Hecht, H. (1992). Lifetime and six-month prevalence of mental disorders in the Munich Follow-Up Study. European Archives of Psychiatry and Clinical Neurosciences 241, 247-258.

Wittchen, H.-U., Nelson, G. B. \& Lachner, G. (1998a). Prevalence of mental disorders and psychosocial impairments in adolescents and young adults. Psychological Medicine 28, 109-126.

Wittchen, H.-U., Lachner, G., Wunderlich, U., Pfister, H. (1998b) Test-retest reliability of the computerized DSM-IV version of the Munich-Composite International Diagnostic Interview (M-CIDI). Social Psychiatry and Psychiatric Epidemiology 33, 568-578.

Wittchen, H.-U., Lieb, R., Schuster, P., Oldehinkel, A. (1999). When is onset? Investigations in early developmental stages of anxiety and depressive disorders. American Journal of Psychiatry (in the press.)

World Health Organization, (1990). Composite International Diagnostic Interview (CIDI). World Health Organization: Geneva. 